\title{
In vitro assessment of the efficiency of the PIM-1 kinase pharmacological inhibitor as a potential treatment for Burkitt's lymphoma
}

\author{
MONA ALSUBAIE ${ }^{1-3^{*}}$, SABINE MATOU-NASRI $^{1,4^{*}}$, ABDULLAH ALJEDAI $^{3 *}$, \\ AHMED ALASKAR ${ }^{4-6}$, HAMAD AL-EIDI $^{1}$, SARAH A. ALBABTAIN $^{7}$, KHAWLAH E. ALDILAIJAN $^{7}$, \\ MANAL ALSAYEGH $^{1}$ and IBRAHIM B. ALABDULKAREEM ${ }^{1,7}$
}

\begin{abstract}
${ }^{1}$ Cell and Gene Therapy Group, Medical Genomics Research Department, King Abdullah International Medical Research Center, Ministry of National Guard-Health Affairs, Riyadh 11481; ${ }^{2}$ Hematology and Serology Unit, Department of Laboratory Medicine Sciences, College of Applied Medical Sciences, Prince Sattam Bin Abdulaziz University, Al-Kharj, Riyadh 11942; ${ }^{3}$ Department of Clinical Laboratory Sciences, College of Applied Medical Sciences, King Saud University, Riyadh 12372; ${ }^{4}$ College of Medicine, King Saud bin Abdulaziz University for Health Sciences, Riyadh 11481; ${ }^{5}$ Division of Adult Hematology and Hematopoietic Stem Cell Transplantation, Department of Oncology, King Abdullah Medical City, Ministry of National Guard-Health Affairs, Riyadh 14611; ${ }^{6}$ King Abdullah International Medical Research Center, Ministry of National Guard-Health Affairs, Riyadh 11426; ${ }^{7}$ Research Department, Health Sciences Research Center, Princess Nourah Bint Abdulrahman University, Riyadh 11564, Saudi Arabia
\end{abstract}

Received November 30, 2020; Accepted February 18, 2021

DOI: $10.3892 / \mathrm{ol} .2021 .12883$

\begin{abstract}
Burkitt's lymphoma is an aggressive form of lymphoma affecting B lymphocytes. It occurs endemically in Africa and sporadically in the rest of the world. Due to the high proliferation rate of this tumor, intensive multi-drug treatment is required; however, the risk of tumor syndrome lysis is high. Overexpression of the proto-oncogene proviral integration of the Moloney murine leukemia virus (PIM-1) kinase is associated with the development of hematological abnormalities, including Burkitt's lymphoma (BL). PIM-1 primarily exerts anti-apoptotic activities through BAD phosphorylation. The aim of the present study was to investigate the in vitro efficiency of a PIM-1 kinase pharmacological inhibitor (PIM1-1) in BL. The impact of PIM1-1 was evaluated in terms of the viability and apoptosis status of the BL B cell lines, Raji and Daudi,
\end{abstract}

\footnotetext{
Correspondence to: Dr Sabine Matou-Nasri or Dr Ibrahim B. AlAbdulkareem, Cell and Gene Therapy Group, Medical Genomics Research Department, King Abdullah International Medical Research Center, Ministry of National Guard Health Affairs, P. O. Box 22490, Riyadh 11481, Saudi Arabia

E-mail: matouepnasrisa@ngha.med.sa

E-mail: alabdulkarym@gmail.com

*Contributed equally
}

Key words: Burkitt's lymphoma, Daudi cells, Raji cells, proviral integration of the Moloney murine leukemia virus kinase, proviral integration of the Moloney murine leukemia virus pharmacological inhibitor, apoptosis compared with K562 leukemia cells, which highly express PIM-1. Cell viability and apoptotic status were assessed with western blotting, and PIM-1 gene expression was assessed with reverse transcription-quantitative PCR. After $48 \mathrm{~h}$ of treatment, PIM1-1 inhibited the Daudi, Raji and K562 cell viability with a half-maximal inhibitory concentration corresponding to 10, 20 and $30 \mu \mathrm{M}$ PIM1-1, respectively. A significant decrease of ERK phosphorylation was detected in PIM1-1-treated Daudi cells, confirming the antiproliferative effect. The addition of $10 \mu \mathrm{M}$ PIM1-1 significantly decreased the PIM-1 protein and gene expression in Daudi cells. An inhibition of the pro-apoptotic BAD phosphorylation was observed in the Daudi cells treated with 0.1-1 $\mu \mathrm{M}$ PIM1-1 and $10 \mu \mathrm{M}$ PIM1-1 decreased BAD phosphorylation in the Raji cells. The apoptotic status of both PIM1-1-treated cells lines were confirmed with the detection of cleaved capase-3. However, no change in cell viability and PIM-1 protein expression was observed in the $10 \mu \mathrm{M}$ PIM1-1-treated K562 cells. In conclusion, the findings indicated that the PIM1-1 pharmacological inhibitor may have therapeutic potential in BL, but with lower efficiency in leukemia.

\section{Introduction}

Burkitt's lymphoma (BL) is an aggressive B cell non-Hodgkin lymphoma (NHL), occurring in three distinct clinical and epidemiological variants: Sporadic, endemic and immunodeficiency-associated forms. The hallmark of BL is the chromosomic translocation $\mathrm{t}(8 ; 14)$, which causes the upregulation of the c-MYC protein transcription factor as well as uncontrolled B cell proliferation, accounting for the rapid growth rate of BL tumor cells (1). c-MYC is stabilized through the phosphorylation function of the human proto-oncogene 
proviral integration of the Moloney murine leukemia virus (PIM-1) serine/threonine kinase, underpinning the critical regulatory role of PIM-1 in the c-MYC-driven BL tumor cell development (2).

The PIM-1 serine/threonine kinase is a highly conserved protein. PIM-1 is expressed in normal lymphoid and myeloid hematopoietic cells and in various human tissues, such as prostate, testis and oral epithelial cells (3). PIM-1 has a constitutive kinase activity that critically regulates cell migration, cell cycle, cell proliferation, cell survival and exerts anti-apoptotic activities (4). Elevated PIM-1 expression predicts a poor outcome in hematological malignancies, including acute myeloid leukemia, B cell chronic lymphocytic leukemia, diffuse large B cell lymphoma and BL (5). PIM-1-overexpression contributes to tumorigenesis through three pathways, including inhibiting apoptosis (i.e., programmed cell death), stimulating cell proliferation and promoting genomic instability (6). For instance, PIM-1 tumorigenic activity has a synergizing effect with c-MYC in lymphomagenesis via the phosphorylation and inactivation of the pro-apoptotic BAD protein, which inhibits the cleavage of the key pro-apoptotic executioner caspase-3 (7). Several studies reported that highly expressed PIM-1 is co-located with c-MYC in the nucleus of BL B cells, which strongly accelerates c-MYC-driven lymphomagenesis $(8,9)$.

Current anticancer therapies available for BL are associated with life-threatening side effects because of tumor lysis syndrome, especially in older patients with poorer outcomes compared with younger patients (10). Chemoresistance has also been detected in tumor cells overexpressing PIM-1, such as BL B cells (11). PIM-1 kinase is important in BL tumorigenesis and chemoresistance and could be a promising therapeutic target for patients diagnosed with BL. There is a need for more specific and less toxic molecular PIM-1-targeted therapy options for patients with BL. The aim of the present study was to determine the pro-apoptotic effect of a PIM-1 kinase pharmacological inhibitor (PIM1-1) in two BL B cell lines, Daudi and Raji cells, compared with the K562 leukemia cell line that has high levels of PIM-1.

\section{Materials and methods}

Cell culture and treatment. The BL (Raji and Daudi) and leukemia (K562) cell lines were purchased from the American Type Culture Collection and authenticated by the supplier. The cells were cultured in complete medium consisting of RPMI-1640 with $2 \mathrm{mM}$ L-glutamine, $100 \mu \mathrm{g} / \mathrm{ml}$ streptomycin, $100 \mathrm{IU} / \mathrm{ml}$ penicillin and supplemented with $10 \%$ fetal calf serum, (all Gibco; Thermo Fisher Scientific Inc.). The cells were seeded in T-75 $\mathrm{cm}^{2}$ flasks and maintained in a humidified incubator under standard conditions $\left(37^{\circ} \mathrm{C} ; 5 \% \mathrm{CO}_{2}\right)$.

PIM1-1 (cat. no. 18/144326; $10 \mathrm{mM}$ stock solution; Tocris Bioscience) was solubilized in DMSO and diluted in RPMI-1640. The cells were exposed to various concentrations $(0.1,1,10,20,30$ and $40 \mu \mathrm{M})$ of PIM1-1 and incubated for $48 \mathrm{~h}$. DMSO $0.4 \%$ and $1 \mu \mathrm{M}$ of the protein kinase inhibitor staurosporine (STS) were used as negative and positive controls, respectively.

Cell viability. B cell lines (Raji and Daudi) and leukemic cell line (K562) $\left(10^{4}\right)$ were seeded in $100 \mu \mathrm{l}$ complete RPMI-1640 medium per well of 96-well plates. The cell treatment was applied as aforementioned and was performed in triplicate. The number of viable cells were determined using the CellTiter-Glo ${ }^{\circledR}$ Luminescent Assay kit (Promega Corporation) and based on the quantification of ATP generated from metabolic reactions, which was indicative of active cells. The amount of ATP produced was proportional to the number of viable cells. The inhibitory concentration (IC)-50 of PIM1-1 that resulted in a 50\% decrease in the viable cell number was also calculated.

$R N A$ extraction and reverse transcription-quantitative PCR (RT-qPCR) analysis. Total RNA was extracted from the untreated and treated cells $\left(1.5 \times 10^{5} / \mathrm{cm}^{2}\right)$ using the PureLink ${ }^{\mathrm{TM}}$ RNA Mini kit (Ambion; Thermo Fisher Scientific, Inc.) according to the manufacturer's protocol. High-quality RNA $(1 \mu \mathrm{g})$ was reverse transcribed to cDNA using an Applied Biosystems $^{\mathrm{TM}}$ High-Capacity cDNA Reverse Transcription kit (Thermo Fisher Scientific, Inc.), according to the manufacturer's protocol. The cDNA was used as the template for the quantitative PCR reaction. RT-qPCR was performed through real-time monitoring of the increase in fluorescence of the SYBR ${ }^{\circledR}$ Green dye (cat. no. 4309155; Qiagen $\mathrm{GmbH}$ ), using the 7900 Fast Real-Time PCR system (Thermo Fisher Scientific, Inc.). The primer pair sequences (Invitrogen; Thermo Fisher Scientific) were used as previously described (12). The thermocycling conditions were as follows: Heat denaturing at $95^{\circ} \mathrm{C}$ for $10 \mathrm{~min}$, then by 40 cycles of denaturing at $95^{\circ} \mathrm{C}$ for $10 \mathrm{sec}$ followed by annealing at $57^{\circ} \mathrm{C}$ for $20 \mathrm{sec}$ and finally extension at $72^{\circ} \mathrm{C}$ for $30 \mathrm{sec}$. The fold-change of PIM-1 RNA expression levels measured by the cycle threshold $(\mathrm{Cq})$ values were calculated and normalized to the expression levels of the housekeeping gene $G A P D H$, according to $2^{-\triangle \Delta C q}$ method (13) as follows: Fold-change $=2^{(-\Delta \Delta \mathrm{Cq})}$ with $\Delta \Delta \mathrm{Cq}=\Delta \mathrm{Cq}$ $\left(P I M-1_{\text {treated }}-\mathrm{GAPDH}_{\text {treated }}\right)-\triangle \mathrm{Cq}\left(P I M-1_{\text {control- }}-G A P D H_{\text {control }}\right)$.

Western blotting. The untreated and treated Daudi, Raji and K562 cells were lysed in Nonidet P40 (NP40) Cell Lysis Buffer composed of $50 \mathrm{mM}$ Tris (pH 7.4), $250 \mathrm{mM} \mathrm{NaCl}, 5 \mathrm{mM}$ EDTA, $50 \mathrm{mM} \mathrm{NaF}, 1 \mathrm{mM} \mathrm{Na}_{3} \mathrm{VO}_{4}, 1 \% \mathrm{NP} 40$ and $0.2 \% \mathrm{NaN}_{3}$ (all Invitrogen; Thermo Fisher Scientific, Inc.). The lysates were separated from the cell debris through centrifugation at $20,000 \mathrm{x} \mathrm{g}$ for $15 \mathrm{~min}$ at $4^{\circ} \mathrm{C}$ and the supernatant was collected. The protein concentrations were quantified using the Qubit ${ }^{\mathrm{TM}}$ Protein Assay kit (Thermo Fisher Scientific, Inc.). Total proteins ( $80 \mu \mathrm{g}$ per lane) were separated using $12 \%$ SDS-PAGE and transferred to a polyvinylidene difluoride membrane as described previously (12). The membranes were blocked for $1 \mathrm{~h}$ with $3 \% \mathrm{BSA}$ at room temperature and probed, overnight at $4^{\circ} \mathrm{C}$, with mouse or rabbit primary monoclonal antibodies $(1: 1,000)$ directed against phosphorylated (p)-BAD (Ser112; cat. no. 5284), total BAD (cat. no. 9239), pro-caspase-3 (cat. no. 14220) and cleaved caspase-3 (cat. no. 9664) procured from Cell Signaling Technology, Inc., p-ERK1 (Tyr204 of ERK1, cat. no. sc-7383), total ERK1 (cat. no. sc-271269), PIM-1 (cat. no. sc-13513) obtained from Santa Cruz Biotechnology, Inc. and GAPDH (cat. no. ab8245) from Abcam. The membranes were washed three times in Tris-buffered saline containing $0.1 \%$ Tween- $20(\mathrm{pH} 7.4)$ and incubated with either goat anti-mouse (1:5,000; cat. no. 170-5047; Bio-Rad 
Inc.) or anti-rabbit (1:5,000; cat. no. 170-6515; Bio-Rad Inc.) secondary antibodies conjugated to horseradish peroxidase or with either infrared fluorescent IRDye ${ }^{\circledR} 680 \mathrm{RD}$-conjugated goat anti-rabbit (1:10,000; cat. no. 926-68071; Li-COR Biosciences) or IRDye ${ }^{\circledR} 800 \mathrm{RD}$-cojugated goat anti-mouse (1:10,000; cat. no. 926-32210; Li-COR Biosciences) secondary antibodies, for $1 \mathrm{~h}$ at room temperature. The bands were visualized by incubating the horseradish peroxidase-stained membranes with enhanced chemiluminescence reagents (Clarity $^{\mathrm{TM}}$ Western ECL Substrate; Bio-Rad Laboratories, Inc.) and scanned with c-DiGit (LI-COR Biosciences). The IRDye stained membranes were scanned using a LI-COR Odyssey ${ }^{\circledR}$ CLx Scanner. The protein expression level was quantified using ImageJ software v.1.46r (http://rsbweb.nih. gov/ij/index.html).

Statistical analysis. All the data are expressed as mean \pm standard deviation and each experiment was independently repeated three times. For comparison between the groups, one-way ANOVA with Tukey's HSD post hoc test was used. SPSS Statistics 64-bit MS Windows v.22.0.0.0 (IBM, Corp.) was used to analyze the data, and for gene expression levels, the Applied Biosystems $^{\text {TM }}$ QuantStudio $^{\text {TM }} 6$ Flex system (Thermo Fisher Scientific, Inc.) was used. $\mathrm{P}<0.05$ was considered to indicate a statistically significant difference.

\section{Results}

PIM1-1 inhibits Raji, Daudi and K562 cell viability. To study the inhibitory effect of PIM1-1 on Raji, Daudi and K562 cell viability, the cells were incubated for $48 \mathrm{~h}$ with different concentrations of PIM1-1 (range, 0.1-40 $\mu \mathrm{M}$ ), with $0.4 \%$ DMSO (negative control) and $1 \mu \mathrm{M} \mathrm{STS} \mathrm{(positive} \mathrm{control).} \mathrm{The}$ untreated and treated cells were subjected to a luminescent cell viability assay using CellTiter-Glo ${ }^{\mathrm{TM}}$. As shown in Fig. 1, a slight increase of Raji and Daudi cell viability was observed at low concentrations of PIM1-1 at 0.1 and $1 \mu \mathrm{M}$; indicating a slightly higher amount of ATP generated by treated cells compared with the ATP amount generated by untreated cells. At higher concentrations, PIM1-1 significantly decreased the Daudi cell viability by $\sim 50 \%$ at $10 \mu \mathrm{M}(\mathrm{P}=0.0028)$ and by $98 \%$ at $40 \mu \mathrm{M}(\mathrm{P}<0.0001)$. In the Raji cells, PIM1-1 significantly decreased cell viability by $50 \%(\mathrm{P}=0.0021)$ at $20 \mu \mathrm{M}$ and by $\sim 85 \%(\mathrm{P}<0.0001)$ at $40 \mu \mathrm{M}$. However, for $\mathrm{K} 562$ cells, a $48.8 \%$ decrease in viability was measured at $30 \mu$ M PIM1-1 ( $\mathrm{P}=0.015)$ and $44.8 \%$ at $40 \mu \mathrm{M}(\mathrm{P}=0.0051)$. Increasing concentrations of PIM1-1 decreased the Raji, Daudi and K562 cell viability in a dose-dependent manner. As estimated from the sigmoidal dose curve with a variable slope, the IC50 for the Daudi, Raji and K562 cells were at 10, 20 and $30 \mu \mathrm{M}$ of PIM1-1, respectively (data not shown). The PIM-1 kinase pharmacological inhibitor PIM1-1 inhibited the cell growth of the BL B cell lines (Raji and Daudi), and the growth of leukemic cell line K562 was the least affected (Fig. 1).

To confirm the decrease of cell proliferation, the level of p-ERK in the untreated cells and cells treated with DMSO, STS or $(0.1,1.0$ or $10 \mu \mathrm{M})$ PIM1-1 were assessed using western blotting. In the Raji cells, any addition of PIM1-1 slightly decreased p-ERK1 compared with the untreated cells (Fig. 2). However, a significant decrease in the level of p-ERK1 was

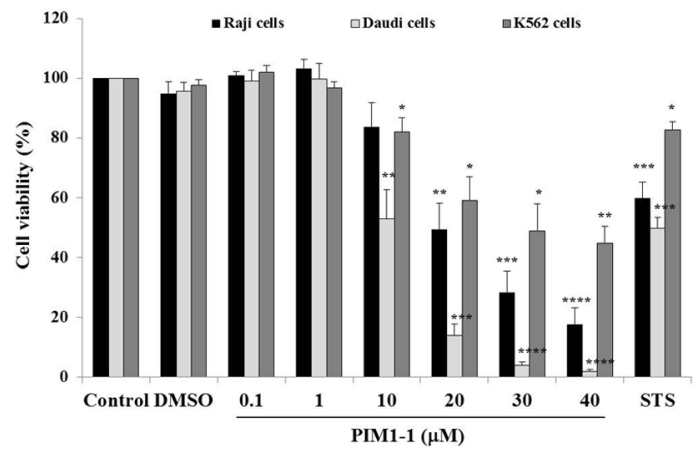

Figure 1. Inhibitory effect of PIM1-1 on Raji, Daudi and K562 cell viability. Cell viability was determined after $24 \mathrm{~h}$ of cell treatment with different concentrations (0.1-40 $\mu \mathrm{M})$ of PIM1-1. Viability of Raji, Daudi and K562 cells decreased when treated with $\geq 10 \mu \mathrm{M}$ of pim1-1 and $1 \mu \mathrm{M}$ STS, the positive control. ${ }^{*} \mathrm{P}<0.05$, ${ }^{* *} \mathrm{P}<0.01,{ }^{* * *} \mathrm{P}<0.001$ and ${ }^{* * * * *} \mathrm{P}<0.0001$ compared with respective untreated cells. DMSO, dimethyl sulfoxide; PIM, proviral integration of the Moloney virus; PIM1-1, PIM-1 kinase pharmacological inhibitor; STS, staurosporine.

observed in the Daudi cells treated with $10 \mu \mathrm{M}$ of PIM1-1 compared with the untreated cells. Used as a positive control, the protein kinase inhibitor STS, when added to Raji and Daudi cells, resulted in a significant decrease of the level of p-ERK1 compared with the p-ERK1 expression level detected in the untreated cells (Fig. 2). No change was observed in the level of p-ERK1 in the K562 cells in all the conditions used. Hence, various antiproliferative effects of PIM1-1 on the BL B cell lines Raji and Daudi were confirmed by the variation of the decrease in the levels of p-ERK1 measured in each PIM1-1-treated B cell line. At the low concentrations at which the PIM1-1 was tested (0.1-10 $\mu \mathrm{M})$, the leukemic cell growth based on p-ERK1 level remained unchanged.

PIM1-1 induces the downregulation of PIM-1 and BAD phosphorylation in the Daudi and Raji cell lines. PIM-1 negatively regulates its own expression (14). PIM1-1 kinase inhibitor efficiency was assessed based on the PIM-1 kinase expression level. At the protein level, using western blotting, PIM1-1 tested between 0.1 and $10 \mu \mathrm{M}$ did not significantly decrease PIM-1 protein expression in PIM1-1-treated Raji cells (Fig. 3). However, at 1 and $10 \mu \mathrm{M}$, PIM1-1 significantly decreased PIM-1 protein expression level by $70 \%(\mathrm{P}<0.01)$ in Daudi cells, compared with the expression level detected in untreated Daudi cells (Fig. 3A). For the K562 cells, PIM1-1 did not change the PIM-1 protein expression level compared with untreated cells (Fig. 3). STS significantly inhibited PIM-1 protein expression level by $85 \%$ in both the Raji and Daudi cells, but not in K562 cells (Fig. 3A). As expected, DMSO did not affect the PIM-1 expression level in all types of cells compared with untreated cells (Fig. 3).

PIM-1 kinase phosphorylates the pro-apoptotic protein BAD and promotes BAD inactivation, which supports cell survival $(7,15)$. The level of p-BAD was evaluated to confirm the variation of PIM-1 kinase expression observed in the PIM1-1-treated cells. Notably, a significant decrease in p-BAD was observed in the Raji cells treated with $10 \mu \mathrm{M}$ PIM1-1 compared with the level detected in the untreated cells (Fig. 3). A significant decrease and quasi-disappearance of p-BAD was observed in Daudi cells treated with 1 and 

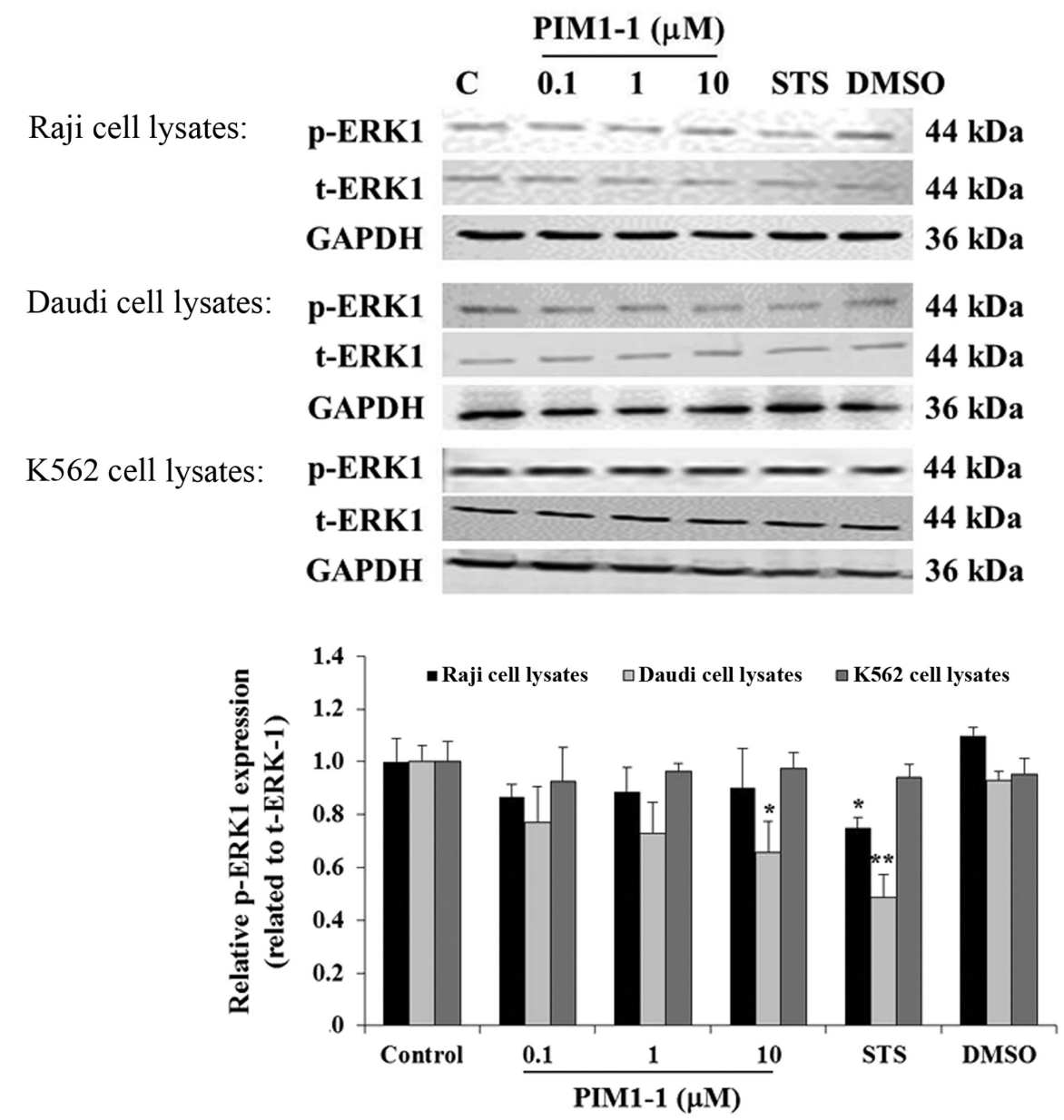

Figure 2. Variations of p-ERK1 expression levels in PIM1-1-treated Raji and Daudi cells. p-ERK1 expression levels in the Raji and Daudi cells following $48 \mathrm{~h}$ treatment with a range of concentrations (range, 0.1-10 $\mu \mathrm{M}$ ) of PIM1-1. Bar graph shows the relative expression levels of p-ERK1, calculated as a ratio of the expression to t-ERK1. "P<0.05 and ${ }^{* *} \mathrm{P}<0.01$ compared with the control. t-, total; p-, phosphorylated; PIM, proviral integration of the Moloney virus; PIM1-1, PIM-1 kinase pharmacological inhibitor.

$10 \mu \mathrm{M}$ of PIM1-1 compared with the untreated cells (Fig. 3). As expected, STS decreased p-BAD levels in both Raji and Daudi cells, but DMSO did not affect p-BAD in the three cell lines (Fig. 3). In all the conditions applied, the level of p-BAD in the K562 cells did not change, compared with the untreated cells (Fig. 3).

To verify the downregulation of the PIM-1 protein expression observed in PIM1-1-treated Daudi cells, PIM-1 mRNA expression level was examined in Daudi cells treated with $10 \mu \mathrm{M}$ PIM1-1. A significant decrease $(\sim 70 \%$; $\mathrm{P}=0.0003)$ of the PIM-1 mRNA expression level was observed in PIM1-1-treated Daudi cells compared with the untreated and DMSO-treated cells (Fig. 4).

Detection of cleaved caspase-3 in PIM1-1-treated Raji and Daudi cells. At the functional level, the blockade of PIM-1 kinase activity principally results in the induction of apoptosis (16). After Raji and Daudi cell treatment with various concentrations (range, $0.1-10 \mu \mathrm{M}$ ) of PIM1-1, the protein extracts were subjected to western blotting for the detection of cleaved caspase-3, a hallmark of apoptosis (17). A weak detection of cleaved caspase-3 was observed in the PIM1-1-treated Raji cells; however, cleaved caspase-3 was observed in the Daudi cells treated with $10 \mu \mathrm{M}$ PIM1-1 (Fig. 5). As expected, no cleavage of pro-caspase-3 was observed in the DMSO-treated cells but some cleavage occurred in STS-treated cells (Fig. 5).

\section{Discussion}

In hematopoietic cells, PIM-1 is involved in the development and function of the cells. The overexpression of PIM-1 kinase is found in the majority of hematological malignancies, including myeloid and lymphoid leukemia and B cell NHL (18). PIM-1-overexpression contributes to malignant cell proliferation and through dysregulation of the cell cycle and inhibition of apoptosis $(19,20)$. Several studies have investigated the effect of PIM-1-downregulation and its impact on cell survival and apoptosis $(16,21)$. The results of the present study established a connection between PIM-1 inhibition using the PIM-1 pharmacological inhibitor, PIM1-1, and the inhibition of cell survival and increased apoptosis, suggesting that targeting PIM-1 kinase is a potentially promising therapeutic approach in BL. Small molecule inhibitors of PIM kinases have been investigated in preclinical and clinical studies to treat hematological and solid cancer types (21-23). The selective PIM1-1 drug investigated in the present study belongs to the pyridone-based family of small molecules inhibitors of PIM-1 kinase which has been shown to exert an inhibitory effect by binding to the ATP-binding site of 

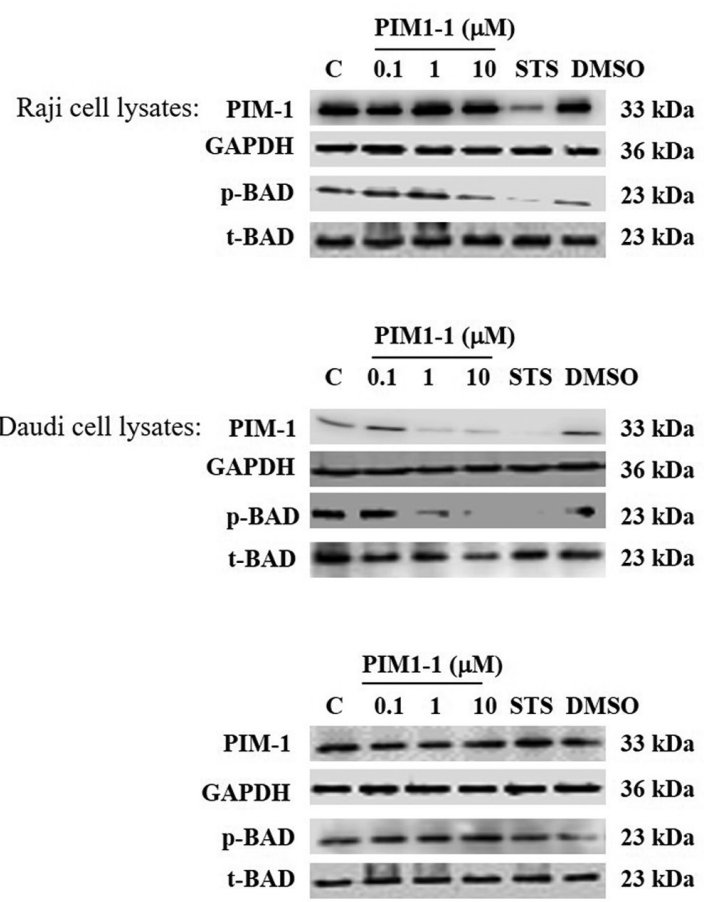
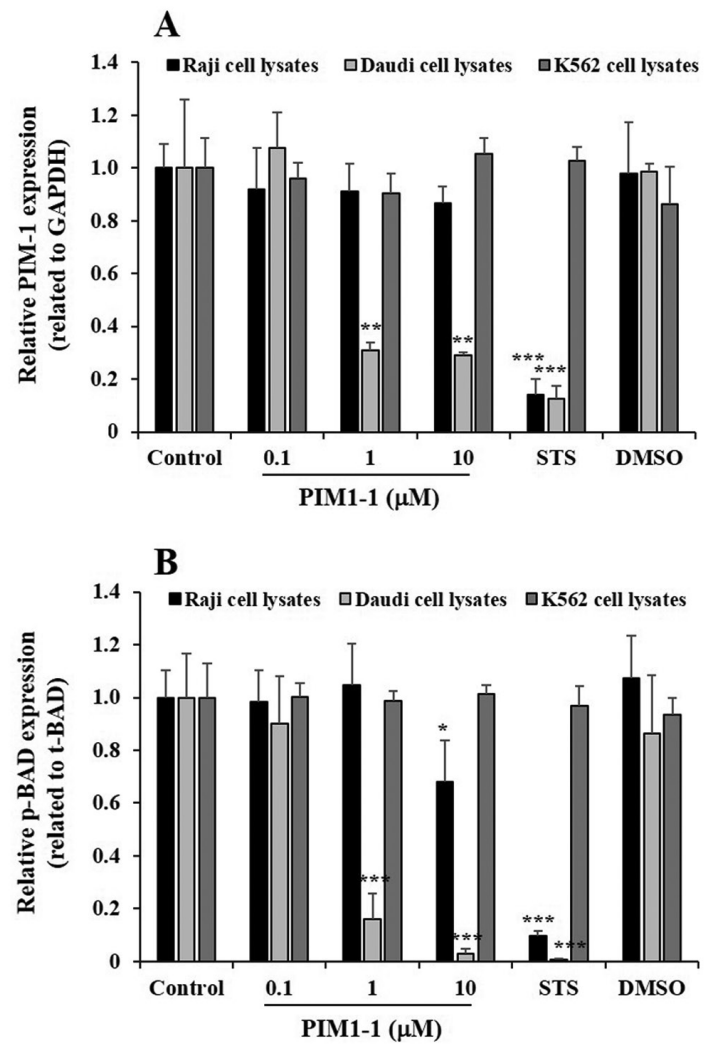

Figure 3. PIM1-1 induces the downregulation of PIM-1 protein expression and decreases phosphorylation of BAD protein in the Raji and Daudi cells. PIM-1 protein expression levels in the Raji, Daudi and K562 cells following $48 \mathrm{~h}$ treatment with a range of concentrations (range, 0.1-10 $\mu \mathrm{M}$ ) of PIM-1.1. Bar graphs show the relative expression levels of the PIM-1 protein (A) and of the p-BAD (B) calculated as a ratio of the expression of the GAPDH and t-BAD loading controls, respectively. ${ }^{*} \mathrm{P}<0.05,{ }^{* *} \mathrm{P}<0.01$ and ${ }^{* * *} \mathrm{P}<0.001$ compared with the control. PIM-1, proviral integration of Moloney murine leukemia virus; p-, phosphorylated-; t-, total-; STS, staurosporine; PIM, proviral integration of the Moloney virus; PIM1-1, PIM-1 kinase pharmacological inhibitor.

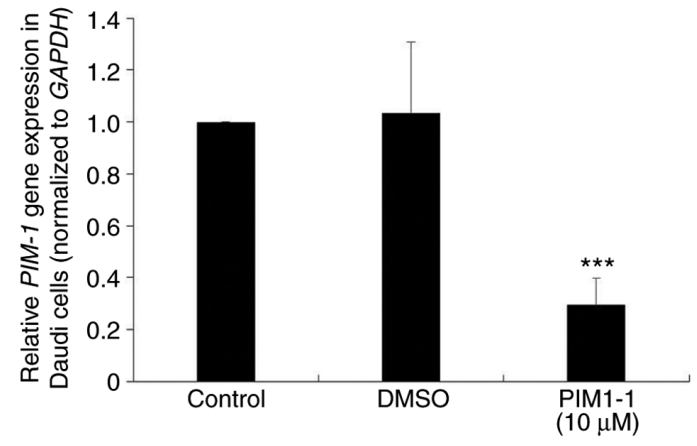

Figure 4. PIM1-1 inhibits PIM-1 gene expression in the Daudi cells. Relative expression level of PIM-1 mRNA was determined by reverse transcription-quantitative PCR analysis in the Daudi cells after 48-h incubation with $10 \mu \mathrm{M}$ of PIM1-1, compared with the untreated and DMSO-treated cells ${ }^{* * *} \mathrm{P}<0.001$ compared with the control. PIM, proviral integration of the Moloney virus; PIM1-1, PIM-1 kinase pharmacological inhibitor.

the PIM-1 kinase, suggesting an ATP-competitive inhibitory mechanism (24). Targeting the kinase action of PIM-1 is expected to prevent the phosphorylation of the downstream effectors and to block its capacity to activate or inactivate proteins involved in cell cycle progression and apoptosis, such as BAD $(7,15)$. In the present study, the treatment of the BL B cell lines, Raji and Daudi, with the novel PIM-1 inhibitor PIM1-1 resulted in decreased cell viability. In addition, a significant decrease of ERK-1 phosphorylation was detected in PIM1-1-treated Daudi cells, confirming the PIM1-1 antiproliferative effect in the BL B cell lines. The current study showed that inhibiting PIM-1 kinase in Daudi and Raji cells with PIM1-1 resulted in a decrease in BAD phosphorylation and induction of apoptosis, revealed by caspase- 3 cleavage. These data highlighted the promising therapeutic potential of PIM1-1 against BL B cell development.

PIM-1 is involved in cell proliferation through the regulation of cell cycle progression and decreased apoptosis $(19,25)$. The current study showed that increasing concentrations of PIM1-1 decreased the Daudi and Raji cell viability in a dose-dependent manner; however, the leukemia cell line K562 was less affected. Based on the IC50 determination, Daudi cells were more sensitive to PIM1-1-inhibition compared with Raji and K562 cells. This observation can be explained by the differential PIM-1-expression levels between both the BL B cell lines and the leukemia cells, with the Daudi cells expressing less PIM-1 compared with the Raji cells (3). The K562 cells, used as a positive control for their high-level protein expression of PIM-1 as previously reported in $(3,12)$, were the least responsive to the PIM1-1 inhibitor. A previous study has shown that quercetagetin (a PIM-1 inhibitor) inhibits cell viability and decreases the colony formation rate of nasopharyngeal carcinoma cells (25). These results are in line with a previous study in which the inhibition of PIM-1 with K00135 [imidazo (1,2-b) pyridazines] in murine $\mathrm{Ba} / \mathrm{F} 3$ cells, acute leukemia cells, and primary blasts from patients with acute myeloid leukemia, selectively reduces cell survival and suppressed the colony proliferation of leukemic blasts (26). In the present study, the decrease in BL B cell line 

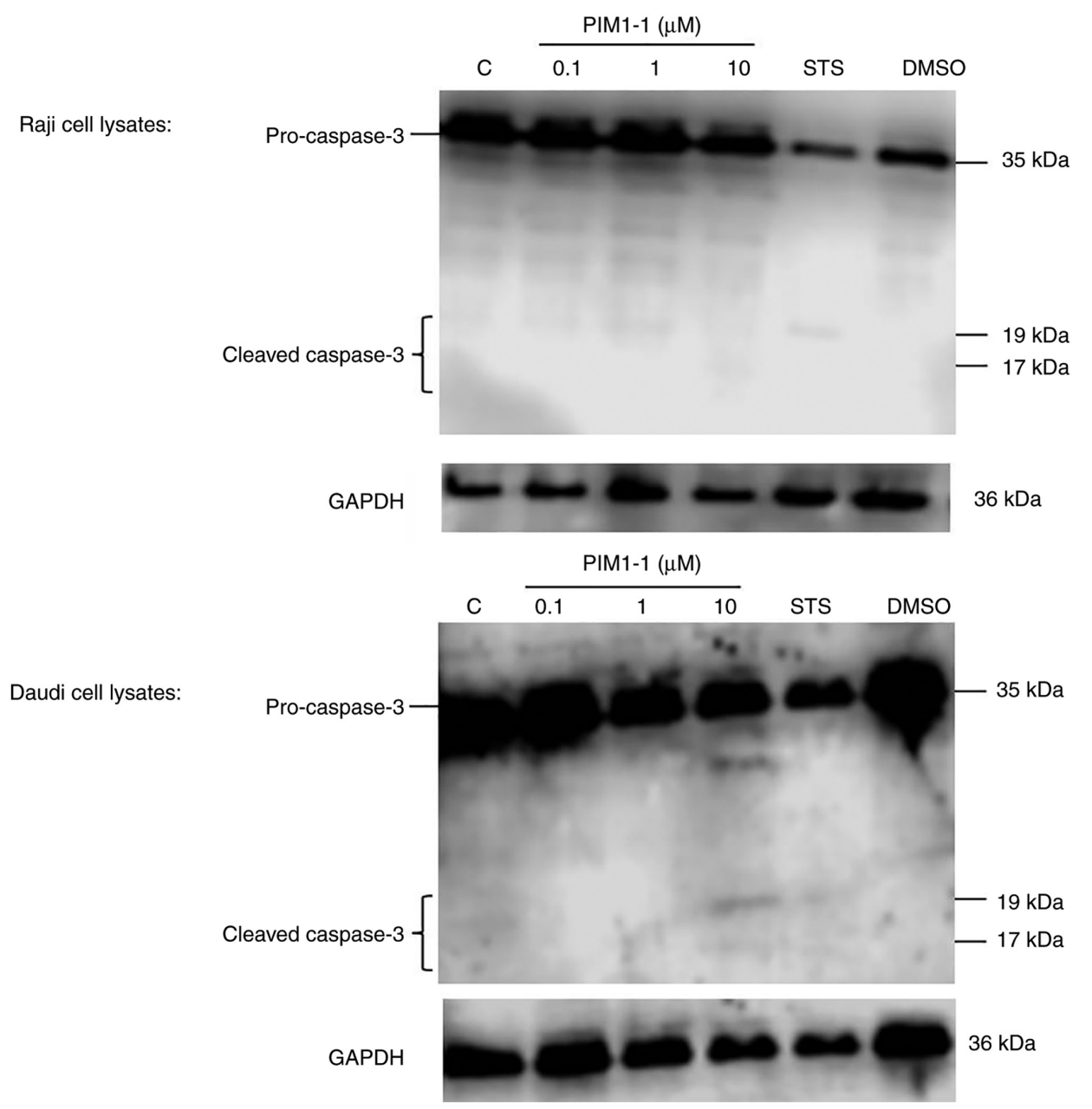

Figure 5. PIM1-1 induces caspase-3 cleavage in the Raji and Daudi cells. Detection of pro-caspase-3 and cleaved caspase-3 in the Raji and Daudi cells following 48-h incubation with a range of concentrations (range, 0.1-10 $\mu \mathrm{M}$ ) of PIM-1.1. Cleaved caspase-3 was not detected in the DMSO-treated cells but was detected in the STS-treated cells. STS, staurosporine; PIM-1, proviral integration of the Moloney virus kinase; PIM1-1, PIM-1 kinase pharmacological inhibitor.

viability caused by PIM1-1 was confirmed by decreased p-ERK, a key signaling protein involved in cell survival and proliferation $(27,28)$. A previous study, conducted with prostate cancer cells, reported that PIM-1-knockdown results in a decrease of p-ERK (29). It is not yet clear whether PIM-1 kinase directly or indirectly affects ERK phosphorylation. However, it would be of interest to investigate the impact of the PIM1-1 kinase inhibitor on substrates with similar regulatory survival pathways, such as ERK and phosphatidylinositol 3-kinase/protein kinase B (PKB also known as Akt) as well $(30,31)$. In addition, Akt/PKB has been demonstrated to be activated in two acute myeloid leukemia cell lines intrinsically resistant to the pan-PIM kinase inhibitor AZD1208, following to elevation of mitochondrial reactive oxygen species (ROS) (32). Furthermore, ROS have been recently reported to be important indicators of drug resistance (33). An assessment of Akt/PKB phosphorylation levels and ROS generated in the BL B cells and leukemic cells could reveal their resistance potential to any PIM-1 kinase inhibitor, including PIM1-1. Altogether, a combination of pharmacological inhibitors targeting survival pathway signaling proteins, ROS production and PIM-1 kinase could enhance the sensitivity of the BL B cells and leukemic cells to cancer therapy.

In addition to acting as a survival factor, PIM-1 kinase also plays a role as both a transcription factor and an activator of several transcription factors, including the signal transducer activator transcription-3, which stimulates PIM-1 gene expression (3). In the current study, $10 \mu \mathrm{M}$ of the PIM1-1 pharmacological inhibitor reduced the PIM-1 mRNA and protein expression levels in the Daudi cells, with no change in the PIM1-1-treated Raji cells compared with the untreated cells. These findings provide evidence for the differential expression of PIM-1 in the Daudi and Raji BL B cell lines, confirming that the Daudi cells are the most responsive to PIM1-1.

PIM1-1 deactivates the pro-apoptotic BAD protein by phosphorylation of Ser112, and changes in the level of this phosphorylation indicates variation of PIM-1 kinase activity (15). $\mathrm{BAD}$ is a pro-apoptotic member of a Bcl-2 group that assists with cell death. The phosphorylation of BAD enhances the binding of BAD to 14-3-3 proteins to block the associations between BAD with the anti-apoptotic proteins $\mathrm{Bcl} 2$ and $\mathrm{Bcl}-\mathrm{xL}$ (34). It has been reported that PIM-1 may play a critical role in the control of survival signaling through the inhibition of mitochondrial pro-apoptotic members of the Bcl-2 family, such as BAD $(15,35)$. In the present study, administration of the PIM1-1 inhibitor caused a significant reduction in the BAD phosphorylation level in the Daudi cells from $1 \mu \mathrm{M}$ of PIM1-1, while the Raji cells showed a reduction on the p-BAD expression level at $10 \mu \mathrm{M}$ of PIM1-1. These findings are similar to those reported by Chen et al (16) in 2011 who 
demonstrated the downregulation of p-BAD in acute myeloid leukemia cells treated with the pan-PIM inhibitor SGI-1776. Forshell et al (9) also reported in 2011 that treating Myc-induced B cell lymphoma with the pan-PIM kinase inhibitor (named as Pim1) caused the dephosphorylation of BAD and the induction of apoptosis. The reduction in PIM-1 expression observed in the current study may have contributed to the decrease in BAD phosphorylation and to the induction of apoptosis, revealed by caspase-3 cleavage. Although executioner caspases, including caspase-3, function in natural cancer cell death (36), PIM-1 phosphorylates the endogenous apoptosis signaling kinase (ASK)1, which suppresses the activation of pro-caspase-3 and maintains cancer cell survival (37). In the present study, inhibition of PIM-1 kinase allowed caspase-3-activation in BL B cell lines, which resulted in strong cleaved caspase- 3 expression in Daudi cells and weak expression in the Raji cells after treatment with 1-10 $\mu$ M PIM1-1. An assessment of ASK1 phosphorylation level using western blotting, expected to decrease following BL B cell treatment with PIM1-1, could confirm apoptosis induction through caspase activation.

In conclusion, the present study demonstrated that the novel PIM1-1 pharmacological inhibitor effectively downregulated PIM-1 expression, markedly decreased cell viability and induced apoptosis, which was revealed by caspase-3 cleavage, in BL B cell lines. These findings provide new evidence for PIM-1 kinase inhibition as a potential therapeutic target for BL treatment. Due to the limited number of cell lines used in the current study, it would be interesting to evaluate the effects of PIM1-1 using other B cell lymphoma cell lines or animal models. Studying the effect of PIM-1-knockdown on other genes involved in cell proliferation, survival, homing, cell signaling, apoptosis and migration is also required. Future studies should examine the effect of PIM1-1 on primary BL cells obtained from patients and to analyze the chromosomal translocation frequency and c-MYC expression levels, which are the main genetic hallmarks of BL (38). In addition, the synergistic effect between PIM1-1 and current chemotherapies should be evaluated to reduce resistance to chemotherapy and to improve the response to the available therapies, which could provide an effective treatment and improve the survival rate for patients diagnosed with BL.

\section{Acknowledgements}

Not applicable.

\section{Funding}

This project was fully funded by King Abdullah International Medical Research Center (grant no. RC12/092).

\section{Availability of data and materials}

The datasets used and/or analyzed during the current study are available from the corresponding author on reasonable request.

\section{Authors' contribution}

IA and AhA conceived the study. IA, SMN and AbA conducted the study. MoA, HAE, SA, KA and MaA conducted the experiments, collected the data, analyzed the data and reviewed the manuscript. Authenticity of all raw data were confirmed by SMN and AbA. MoA, SMN, AbA, AhA and IA wrote the manuscript. All authors read and approved the final version of the manuscript.

\section{Ethics approval and consent to participate}

Not applicable.

\section{Patient consent for publication}

Not applicable.

\section{Competing interests}

The authors declare that they have no competing interests.

\section{References}

1. Kalisz K, Alessandrino F, Beck R, Smith D, Kikano E, Ramaiya NH and Tirumani SH: An update on burkitt lymphoma: A review of pathogenesis and multimodality imaging assessment of disease presentation, treatment response, and recurrence. Insights Imaging 10: 56, 2019.

2. Tursynbay Y, Zhang J, Li Z, Tokay T, Zhumadilov Z, Wu D and Xie Y: Pim-1 kinase as cancer drug target: An update. Biomed Rep 4: 140-146, 2016.

3. Bachmann M and Möröy T: The serine/threonine kinase Pim-1. Int J Biochem Cell Biol 37: 726-730, 2005.

4. Mondello P, Cuzzocrea S and Mian M: Pim kinases in hematological malignancies: Where are we now and where are we going? J Hematol Oncol 7: 95, 2014.

5. Panchal NK and Sabina EP: A serine/threonine protein PIM kinase as a biomarker of cancer and a target for anti-tumor therapy. Life Sci 255: 117866, 2020.

6. Magnuson NS, Wang Z, Ding G and Reeves R: Why target PIM1 for cancer diagnosis and treatment? Future Oncol 6: 1461-1478, 2010.

7. Macdonald A, Campbell DG, Toth R, McLauchlan H, Hastie CJ and Arthur JS: Pim kinases phosphorylate multiple sites on Bad and promote 14-3-3 binding and dissociation from Bcl-XL. BMC Cell Biol 7: 1, 2006.

8. Ionov Y, Le X, Tunquist BJ, Sweetenham J, Sachs T, Ryder J, Johnson T, Lilly MB and Kraft AS: Pim-1 protein kinase is nuclear in Burkitt's lymphoma: Nuclear localization is necessary for its biologic effects. Anticancer Res 23: 167-178, 2003.

9. Forshell LP, Li Y, Forshell TZ, Rudelius M, Nilsson L, Keller U and Nilsson J: The direct Myc target Pim3 cooperates with other Pim kinases in supporting viability of Myc-induced B-cell lymphomas. Oncotarget 2: 448-460, 2011.

10. McBride A, Trifilio S, Baxter N, Gregory TK and Howard SC: Managing tumor lysis syndrome in the era of novel cancer therapies. J Adv Pract Oncol 8: 705-720, 2017.

11. Zemskova M, Sahakian E, Bashkirova S and Lilly M: The PIM1 kinase is a critical component of a survival pathway activated by docetaxel and promotes survival of docetaxel-treated prostate cancer cells. J Biol Chem 283: 20635-20644, 2008.

12. Matou-Nasri S, Rhaban Z, Al-Baijan H, Al-Eidi H, Yahya WB, Al Abdulrahman A, Almobadel N, Alsubeai M, Al Ghamdi S, Alaskar A, et al: CD95-mediated apoptosis in Burkitt's lymphoma $\mathrm{B}$-cells is associated withPim-1 down-regulation. Biochim Biophys Acta Mol Basis Dis 1863: 239-252, 2017.

13. Livak KJ and Schmittgen TD: Analysis of relative gene expression data using real-time quantitative PCR and the 2(-Delta Delta C(T)) method. Methods 25: 402-408, 2001.

14. Peltola KJ, Paukku K, Aho TL, Ruuska M, Silvennoinen O and Koskinen PJ: Pim-1 kinase inhibits STAT5-dependent transcription via its interactions with SOCS1 and SOCS3. Blood 103: 3744-3750, 2004

15. Aho TL, Sandholm J, Peltola KJ, Mankonen HP, Lilly M and Koskinen PJ: Pim-1 kinase promotes inactivation of the pro-apoptotic Bad protein by phosphorylating it on the Ser112 gatekeeper site. FEBS Lett 571: 43-49, 2004. 
16. Chen LS, Redkar S, Taverna P, Cortes JE and Gandhi V: Mechanisms of cytotoxicity to Pim kinase inhibitor, SGI-1776, in acute myeloid leukemia. Blood 118: 693-702, 2011.

17. Elmore S: Apoptosis: A review of programmed cell death. Toxicol Pathol 35: 495-516, 2007.

18. Shah N, Pang B, Yeoh KG, Thorn S, Chen CS, Lilly MB and Salto-Tellez M: Potential roles for the PIM1 kinase in human cancer-a molecular and therapeutic appraisal. Eur J Cancer 44 2144-2151, 2008

19. Morishita D, Katayama R, Sekimizu K, Tsuruo T and Fujita N: Pim kinases promote cell cycle progression by phosphorylating and down-regulating $\mathrm{p} 27 \mathrm{Kip} 1$ at the transcriptional and posttranscriptional levels. Cancer Res 68: 5076-5085, 2008.

20. Ouhtit A, Gupta I, Muzumdar S, Shanmuganathan S and Tamimi Y: Understanding the functional discrepancy of Pim-1 in cancer. Front Biosci (Elite Ed) 7: 208-214, 2015.

21. Luszczak S, Kumar C, Sathyadevan VK, Simpson BS, Gately KA Whitaker HC and Heavey S: PIM kinase inhibition: Co-targeted therapeutic approaches in prostate cancer. Signal Transduct Target Ther 5: 7, 2020.

22. Blanco-Aparicio $\mathrm{C}$ and Carnero A: Pim kinases in cancer: Diagnostic, prognostic and treatment opportunities. Biochem Pharmacol 85: 629-643, 2013.

23. Zhang X, Song M, Kundu JK, Lee MH and Liu ZZ: PIM kinase as an executional target in cancer. J Cancer Prev 23: 109-116, 2018.

24. Cheney IW, Yan S, Appleby T, Walker H, Vo T, Yao N, Hamatake R, Hong Z and Wu JZ: Identification and structure-activity relationships of substituted pyridones as inhibitors of Pim-1 kinase. Bioorg Med Chem Lett 17: 1679-1683, 2007.

25. Jie W, He QY, Luo BT, Zheng SJ, Kong YQ, Jiang HG, Li RJ, Guo JL and Shen ZH: Inhibition of Pim-1 attenuates the proliferation and migration in nasopharyngeal carcinoma cells. Asian Pac J Trop Med 5: 645-650, 2012.

26. Pogacic V, Bullock AN, Fedorov O, Filippakopoulos P, Gasser C Biondi A, Meyer-Monard S, Knapp S and Schwaller J: Structural analysis identifies imidazo[1,2-b]pyridazines as PIM kinase inhibitors with in vitro antileukemic activity. Cancer Res 67: 6916-6924, 2007.

27. Lu Z and Xu S: ERK1/2 MAP kinases in cell survival and apoptosis. IUBMB Life 58: 621-631, 2006.
28. Lee S, Rauch J and Kolch W: Targeting MAPK signaling in cancer: Drug resistance and sensitivity. Int J Mol Sci 21: 1102, 2020

29. Wang J, Anderson PD, Luo W, Gius D, Roh M and Abdulkadir SA: Pim 1 kinase is required to maintain tumorigenicity in MYC-expressing prostate cancer cells. Oncogene 31: 1794-1803, 2012.

30. Aziz AR, Farid S, Qin K, Wang H and Liu B: PIM kinases and their relevance to the PI3K/Akt/mTOR pathway in the regulation of ovarian cancer. Biomolecules 8: 7, 2018.

31. Warfel NA and Kraft AS: PIM kinase (and Akt) biology and signaling in tumors. Pharmacol Ther 151: 41-49, 2015.

32. Brunen D, García-Barchino MJ, Malani D, Basheer NJ, Lieftink C, Beijersbergen RL, Murumägi A, Porkka K, Wolf M, Zwaan CM, et al: Intrinsic resistance to PIM kinase inhibition in AML through p38 $\alpha$-mediated feedback activation of mTOR signaling. Oncotarget 7: 37407-37419, 2016.

33. Bhardwaj V and He J: Reactive oxygen species, metabolic plasticity, and drug resistance in cancer. Int J Mol Sci 21: 3412, 2020.

34. Zha J, Harada H, Yang E, Jockel J and Korsmeyer SJ: Serine phosphorylation of death agonist BAD in response to survival factor results in binding to 14-3-3 Not BCL-X(L). Cell 87: 619-628, 1996.

35. Lilly M, Sandholm J, Cooper JJ, Koskinen PJ and Kraft A: The PIM-1 serine kinase prolongs survival and inhibits apoptosis-related mitochondrial dysfunction in part through a bcl-2-dependent pathway. Oncogene 18: 4022-4031, 1999.

36. McIlwain DR, Berger T and Mak TW: Caspase functions in cell death and disease. Cold Spring Harb Perspect Biol 5: a008656, 2013.

37. Gu JJ, Wang Z, Reeves R and Magnuson NS: PIM1 phosphorylates and negatively regulates ASK1-mediated apoptosis. Oncogene 28: 4261-4271, 2009.

38. Nguyen L, Papenhausen P and Shao H: The role of c-MYC in B-cell lymphomas: Diagnostic and molecular aspects. Genes (Basel) 8: 116, 2017.

(i) (3) This work is licensed under a Creative Commons Attribution-NonCommercial-NoDerivatives 4.0 International (CC BY-NC-ND 4.0) License. 